\title{
A dedicated decay-spectroscopy station for the collinear resonance ionization experiment at ISOLDE
}

\author{
M.M. Rajabali ${ }^{\mathrm{a}, *, 1}$, K.M. Lynch ${ }^{\mathrm{b}, \mathrm{c}}$, T.E. Cocolios $^{\mathrm{c}}$, J. Billowes ${ }^{\mathrm{b}}$, M.L. Bissell ${ }^{\mathrm{a}}$, S. De Schepper ${ }^{\mathrm{a}}$, K. Dewolf ${ }^{\mathrm{a}}$, \\ K.T. Flanagan ${ }^{\text {b }}$, F. Le Blanc ${ }^{\text {d }}$, B.A. Marsh ${ }^{\text {e }}$, P.J.R. Mason ${ }^{\text {f }}$, I. Matea ${ }^{\text {d }}$, G. Neyens ${ }^{\text {a }}$, J. Papuga ${ }^{\text {a }}$, T.J. Procter ${ }^{\text {b }}$, \\ S. Rothe ${ }^{\mathrm{e}, \mathrm{g}}$, G.S. Simpson ${ }^{\mathrm{h}}$, A.J. Smith ${ }^{\mathrm{b}}$, H.H. Stroke ${ }^{\mathrm{i}}$, D. Verney ${ }^{\mathrm{d}}$, P.M. Walker ${ }^{\mathrm{f}}$, K. Wendt ${ }^{\mathrm{g}}$, R.T. Wood ${ }^{\mathrm{f}}$ \\ a Instituut voor Kern- en Stralingsfysica, KU Leuven, B-3001 Leuven, Belgium \\ ${ }^{\mathrm{b}}$ School of Physics and Astronomy, The University of Manchester, Manchester M13 9PL, United Kingdom \\ c Physics Department, CERN, CH-1211 Geneva 23, Switzerland \\ d Institut de Physique Nucléaire, CNRS-IN2P3/Université Paris-Sud 11, Orsay, France \\ e Engineering Department, CERN, CH-1211 Geneva 23, Switzerland \\ ${ }^{\mathrm{f}}$ Department of Physics, University of Surrey, Guildford GU2 7XH, United Kingdom \\ ${ }^{\mathrm{g}}$ Institut für Physik, Johannes Gutenberg-Universität Mainz, D-55128 Mainz, Germany \\ ${ }^{\mathrm{h}}$ LPSC, F-38026 Grenoble, France \\ i Department of Physics, New York University, New York, NY 10003, USA
}

\section{A R T I C L E I N F O}

\section{Article history:}

Received 9 October 2012

Received in revised form

18 December 2012

Accepted 18 December 2012

Available online 28 December 2012

Keywords:

Alpha decay

Fission products

$\gamma$-Ray

Laser-assisted decay spectroscopy

\begin{abstract}
A B S T R A C T
A new decay-spectroscopy station (DSS) has been developed to be coupled to the collinear resonance ionization spectroscopy (CRIS) beam line at CERN-ISOLDE. The system uses a rotatable wheel with ten $20 \mu \mathrm{g} / \mathrm{cm}^{2}$ carbon foils as beam implantation sites for the efficient measurement of charged decay products. Silicon detectors are placed on either side of the carbon foil in an optimal geometry to cover a large solid angle for detecting these charged particles. In addition to the silicon detectors at the onbeam axis position, a second pair of off-beam axis detectors are placed at the wheel position $108^{\circ}$ away, allowing longer-lived species to be studied. Up to three high purity germanium detectors can be placed around the chamber for particle-gamma correlated measurement. The radioactive beam is transported through the CRIS beam line before implantation into a carbon foil at the DSS. All materials used in the DSS are UHV-compatible to maintain high vacuum conditions required by the CRIS beam line. This paper describes the DSS and presents the first data collected at the setup during the commissioning run with ${ }^{221} \mathrm{Fr}$.
\end{abstract}

(c) 2012 Elsevier B.V. All rights reserved.

\section{Introduction}

Experiments developed for the study of nuclear properties of rare isotopes produced using ISOL targets are often hindered by the presence of large isobaric contaminants in the beam. This has driven the development of several beam purification techniques of which the laser ion source (LIS) has proved to be very successful [1-4]. Further steps can be taken to reduce the isobaric contamination from surface ionization as described in Refs. [5,6]. Despite these efforts, experiments designed to study rare isotopes that are produced with lower yields may require even further purification of the beam so as to obtain precise decay branching ratios or for the purpose of total absorption spectrometry.

\footnotetext{
* Corresponding author.

E-mail address: mmr.kul@gmail.com (M.M. Rajabali).

${ }^{1}$ Current address: TRIUMF, Vancouver BC, Canada V6T 2 A3.
}

A compelling problem is that of low-lying isomers that cannot easily be distinguished from their ground state using conventional decay spectroscopy or mass separation techniques.

To study cases where additional beam purification becomes essential, a different but complementary method is needed. Insource laser spectroscopy has previously been used for isomer selection and identification [7,8]. Following this selection, experiments such as Coulomb excitation [9] and mass measurements [10] have been performed on isomeric beams. These experiments did suffer from the presence of isobaric contamination, as well as significant ground-state contamination due to the overlap of the Doppler-broadened hyperfine resonances of each isomer [11].

A new experimental set-up has been constructed at ISOLDECERN for performing collinear resonance ionization spectroscopy (CRIS) measurements [12,13]. This method combines the high efficiency and selectivity, commonly achieved in a LIS resonant ionization spectroscopy (RIS) experiment [1] with the high resolution of a collinear laser spectroscopy experiment [11]. 
While initially developed for hyperfine structure studies, this setup will provide ultra-pure beams of rare isotopes and isomers for decay spectroscopy.

A UHV compatible decay spectroscopy station (DSS) has been developed, dedicated to conduct laser assisted decay spectroscopy at the end of the CRIS beam line. The technique utilizes the selectivity of RIS to perform decay measurements on rare isotopes with the ability to distinguish states that are isomeric ( $>1 \mathrm{~ms}$ ) from the ground state. This paper reports on the technical aspect of the DSS where ${ }^{221} \mathrm{Fr}$ was used as part of the commissioning process.

\section{DSS design}

The experimental constraints imposed by the CRIS technique require the DSS to operate at a pressure below $1 \times 10^{-9}$ mbar. This vacuum requirement makes it essential to have components that do not outgas and that can sustain baking temperatures. For this reason, the cables and detectors used in the DSS are of UHV grade material and all the components are made of either stainless steel or polyether ether ketone (PEEK) to reduce out gassing.

\subsection{Windmill design for $\alpha$-decay spectroscopy}

The decay spectroscopy station consists of a rotatable wheel implantation system as shown in Fig. 1(a) and (b). It is based on the design from KU Leuven [14-17] shown in Fig. 1 of Ref. [17], which has provided results in a number of successful experiments $[8,17,18]$. The wheel holds 10 carbon foils, which were produced at the GSI target laboratory [19], with a thickness of $20 \mu \mathrm{g} / \mathrm{cm}^{2} \pm 5 \%$ (90 nm) into which the ion beam is implanted. The carbon foils and the wheel system are favored over a Mylar tape system for the compact size and, more importantly, the better detection efficiency resulting from the improved transmission of $\alpha$ particles, following the decay of implanted ions in the carbon foils.

A stepper motor (17HS-240E, $2.3 \mathrm{~A} /$ phase) is used to rotate the wheel. The controller for the motor is operated via a programmable computer software package. The rotation of the wheel can be controlled using optical switches or by predefining the number of steps that the motor should turn. In order to maintain UHV, the motor is situated externally and connected to the internal windmill system via a magnetic coupling supplied by UHV Design. This coupling has negligible influence on the noise introduced to the signal between the detector and the preamplifier.

Two Canberra passivated implanted planar silicon (PIPS) detectors for particle detection ( $\alpha$, electron, fission fragments) are situated on either side of the implantation carbon foil, as shown in Fig. 1(a). One PIPS detector (Model: BKA 300-17 AM, thickness $300 \mu \mathrm{m}$ ) sits behind the carbon foil and another annular PIPS (APIPS) (Model: BKANPD 300-18 RM, thickness $300 \mu \mathrm{m}$, with an aperture of $4 \mathrm{~mm}$ ) is placed in front of the carbon foil. The detectors are connected to charge sensitive preamplifiers (Canberra model 2003BT) via custom-made Kapton cables and a UHV type-C sub-miniature electrical feed-through. The ion beam is directed through the $4 \mathrm{~mm}$ aperture of the annular detector and implanted into the carbon foil. Decay products from the carbon foil can be measured in either the PIPS or APIPS detectors. In addition to the on-axis detectors, two PIPS detectors can be placed off-axis. Rotating the implanted foil from the on-axis to off-axis detector position allows the measurement of unperturbed decay curves on longer-lived decay products. For $\gamma$-ray detection, up to three high-purity germanium (HPGe) detectors can be placed around the implantation site. In addition to the silicon and germanium detectors, the spectroscopy chamber also houses a microchannel plate (MCP) detector (Hamamatsu F4655-13) for hyperfine structure measurements with the CRIS experiment [13]. The MCP is set up as a negative ion counter. In this configuration, the beam strikes the surface of a copper plate (Fig. 1(c)) and emits secondary electrons which are then electrostatically guided to the MCP resulting in the observed signal.

\subsection{Beam tuning to the carbon foil}

Resonantly ionized bunched beams from the interaction region in the CRIS beam line are deflected to the decay spectroscopy station by applying voltage to a pair of vertical electrostatic plates. The deflected ion beam is transported along the length of a $1.93 \mathrm{~m}$ long beam line before being implanted into a carbon foil in the windmill. In the process, the beam has to be directed through a $64.9 \mathrm{~mm}$ spacing between the copper plate and MCP (see Fig. 1(c)) in addition to being focused through a $4 \mathrm{~mm}$ aperture of the annular detector. To achieve this, ion optics components have been added to the beam line as shown in Fig. 1(c). After the $20^{\circ}$ deflection, the beam is first directed through a quadrupole doublet which corrects for astigmatism introduced earlier in the beam line. It is then passed through a final pair of vertical and horizontal steering plates (see Fig. 1(c)) which along with the quadrupole doublet allow the focus and position of the beam to be optimized for either the copper plate or the carbon foil.

Two sets of collimators are installed in the detection chamber. The first is attached to a manual linear actuator with a series of 3 , 6 and $10 \mathrm{~mm}$ apertures and is housed in the same region as the $\mathrm{MCP}$ detector. The second is a fixed aluminum disk placed in front of the APIPS detector (see Fig. 1(b)) to protect against direct ion implantation into the silicon wafer. The PEEK holder (see Fig. 1(c)) electrically isolates this disk from the grounded support structure as well as the APIPS detector. An electrical contact is made to read the current when an ion beam strikes this collimator enabling its use as a beam tuning device. The beam focus can be monitored during implantation into one of the carbon foils by measuring how much of the beam is lost on the aluminum disk. When it is not in use as a Faraday cup, the collimator is grounded to avoid charge build-up.

To further improve the beam tuning capability to the ionimplantation site, a copper plate (thickness $0.5 \mathrm{~mm}$, diameter $10 \mathrm{~mm}$ ) is installed at the position of one of the carbon foils in the steel wheel of the DSS. The plate is electrically isolated from the steel wheel by two PEEK rings and connected by a spot-welded Kapton cable to a freely rotatable BNC connection installed at the center of the wheel.

\subsection{Shielding and efficiency}

Measurements of the $\gamma$-ray attenuation by the stainless steel body of the chamber and the steel windmill were carried out using a 70\% high purity germanium detector (Model: GC7020). The results are presented in Fig. 2. Known $\gamma$-ray sources of ${ }^{241} \mathrm{Am}$, ${ }^{152} \mathrm{Eu}$ and ${ }^{60} \mathrm{Co}$ were positioned $65 \mathrm{~mm}$ from the detector, with and without the chamber present. More than $92 \%$ of the $59 \mathrm{keV}$ $\gamma$-rays and $52 \%$ of the $123 \mathrm{keV} \gamma$-rays were attenuated (see Fig. 2) by the $3 \mathrm{~mm}$ thick steel walls. At energies above $591 \mathrm{keV}$, fewer than $18 \%$ of the $\gamma$-rays were affected by the steel wall.

The attenuation of the $\gamma$-rays by the steel wheel of the rotatable windmill system was also investigated. Two sources, ${ }^{60} \mathrm{Co}(2.156 \mathrm{kBq})$ and ${ }^{152} \mathrm{Eu}(1.249 \mathrm{kBq})$, were attached to the wheel separated by $108^{\circ}$. Measurements were taken with one source placed in front of the detector while the other was $108^{\circ}$ away. The $\gamma$-ray energy spectra obtained are shown in Fig. 3. 
(a)

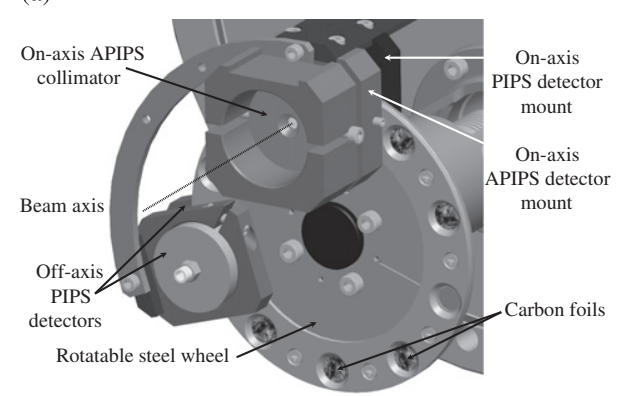

(b)

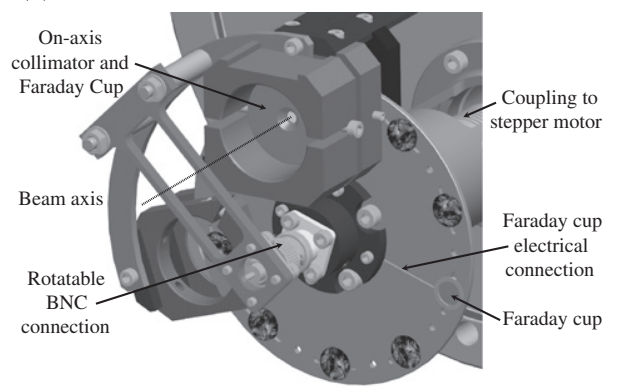

(c)

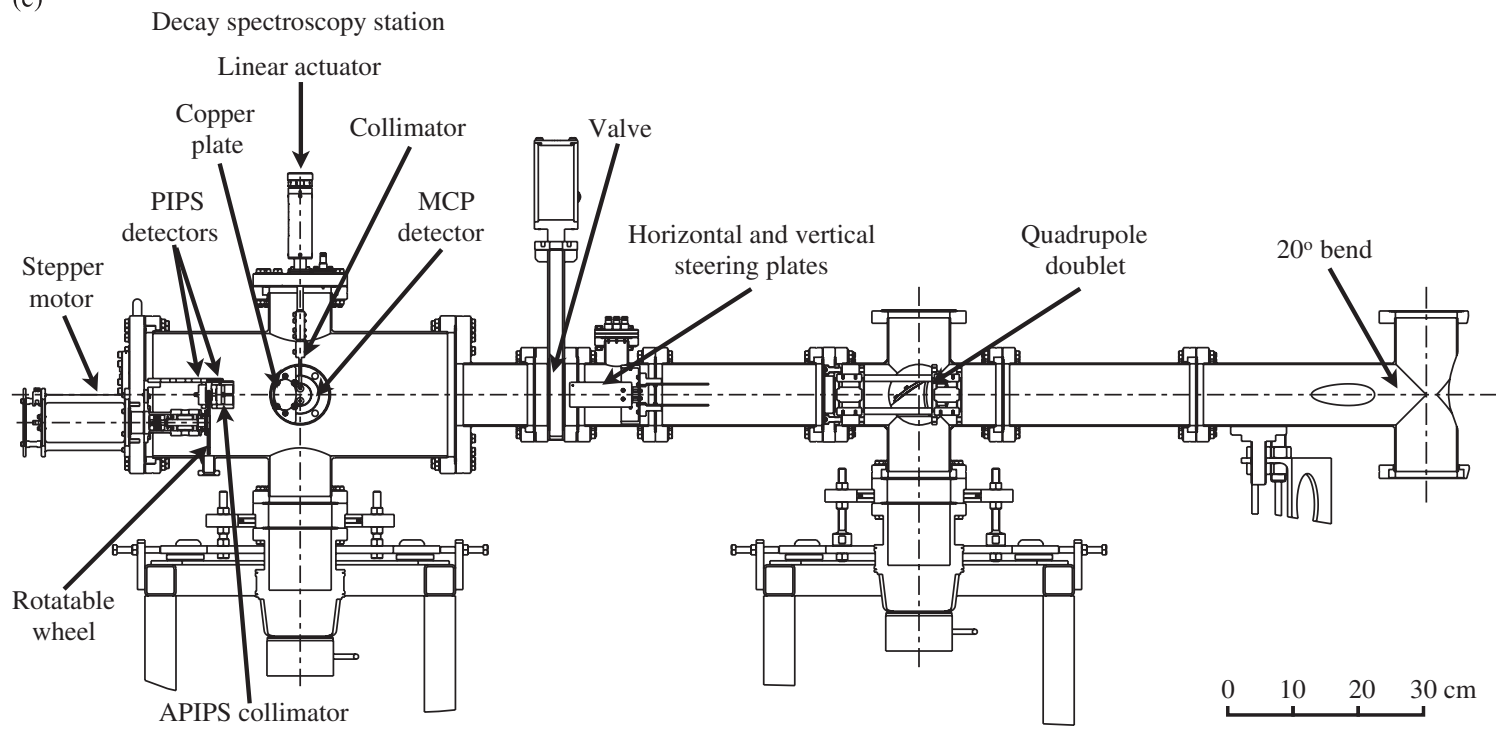

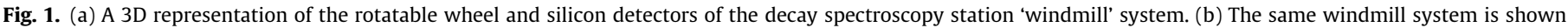

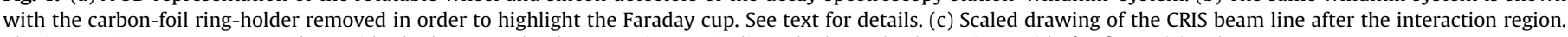
The ion optics components used to guide the beam to the decay station are indicated. The scale shown is a guide for figure (c) only.

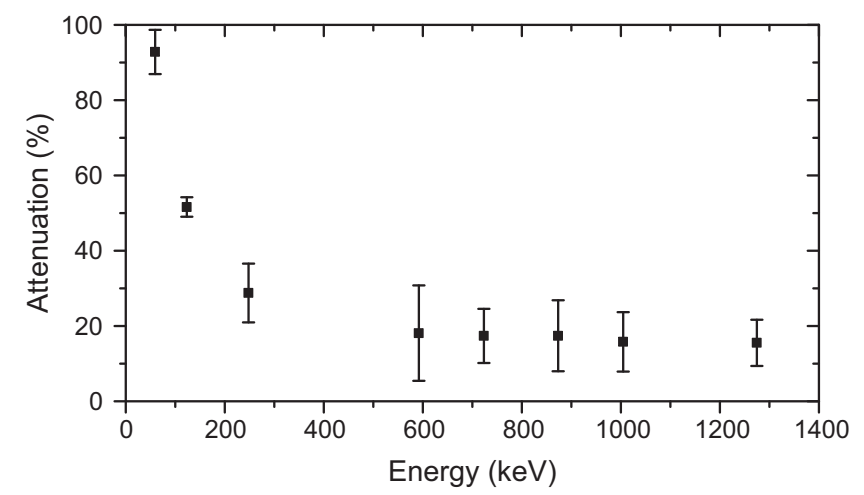

Fig. 2. The percentage of $\gamma$-rays attenuated by the steel wall of the decay spectroscopy station chamber. Lower energy $\gamma$-rays are almost completely attenuated by the steel chamber wall with a loss of $92 \%$ of the $59 \mathrm{keV}$ transition from ${ }^{241} \mathrm{Am}$. Those of higher energy are only partially affected, with a loss of less than $18 \%$ above $591 \mathrm{keV}$.

The black spectrum shows the ${ }^{60}$ Co source directly in front of the germanium detector and the ${ }^{152} \mathrm{Eu}$ source at a position $108^{\circ}$ furthest away from the germanium detector. The red (grey) spectrum shows the ${ }^{152} \mathrm{Eu}$ source in front of the detector and the ${ }^{60} \mathrm{Co}$ at the $108^{\circ}$ position. From Fig. $3(\mathrm{~b})$, it can be seen that the characteristic ${ }^{60} \mathrm{Co}$ lines are decreased by a factor of 10 at $1.3 \mathrm{MeV}$ when the ${ }^{60} \mathrm{Co}$ source is rotated away from the germanium detector. The change in solid angle coverage between the two positions accounts for $\sim 70 \%$ of this reduction. The remaining $\sim 30 \%$ is the result of $\gamma$-ray attenuation by the steel wheel. This provides a shielding advantage for the implanted ions in an off-axis foil when studying decays at the on-beam axis station and vice versa.

The PIPS detector is placed at a distance of $1 \mathrm{~mm}$ from the carbon foil to maximize the solid angle coverage at $45 \%$ (see Fig. 4). The APIPS detector is positioned so as to maximize the solid angle of detection while minimizing the solid-angle loss through the aperture. The optimal distance between the annular PIPS detector and the carbon foil was calculated to be between 3.9 and $5 \mathrm{~mm}$, which corresponds to a solid angle coverage of 16.516.7\%, as shown in Fig. 4.

\subsection{Data acquisition}

The DSS data is acquired with a fully digital data acquisition system (DAQ). This DAQ consist of the XIA digital gamma finder (DGF) revision D modules [20]. Each module has four input channels with a sampling rate of $40 \mathrm{MHz}$. Signals from the silicon and germanium detector preamplifiers are fed into separate modules on account of the different decimation needed to read in the pulses of different temporal length. Decimation in these DGF modules correspond to an averaging of sampling points (25 ns apart) in increments of $2^{x}$ [20]. Signals from the silicon detector preamplifiers are faster than those from the germanium preamplifiers therefore decimation of 2 (100 ns) and 4 (400 ns) are used, respectively. All signals fed into the DAQ are self- 
(a)

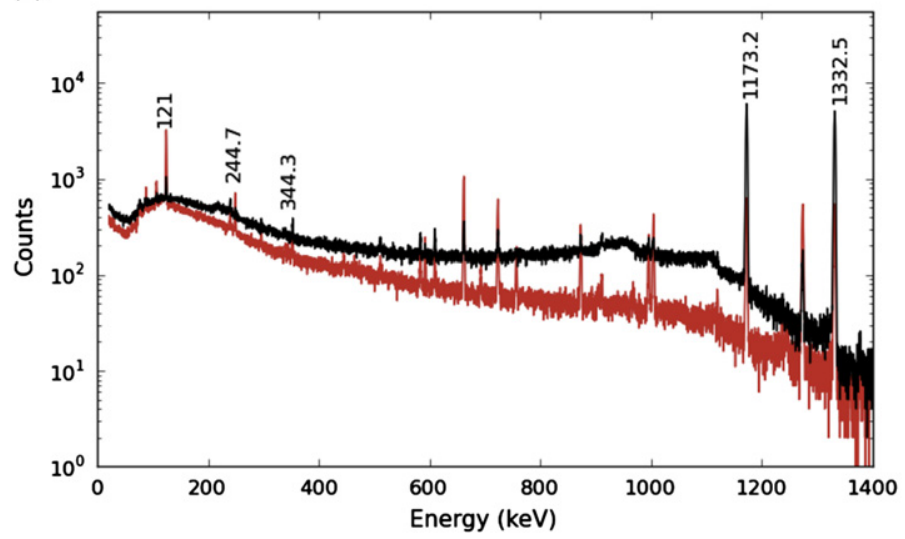

(b)

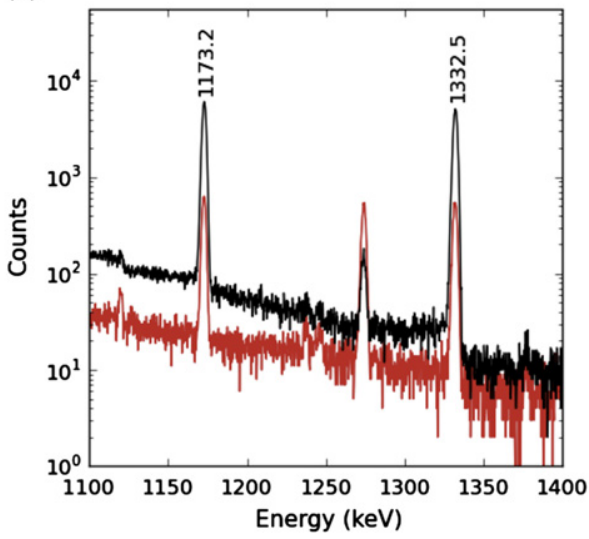

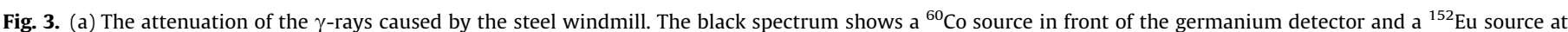

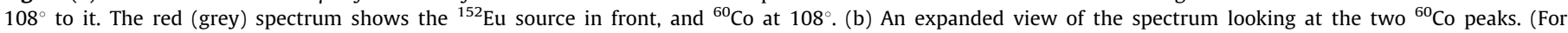
interpretation of the references to color in this figure legend, the reader is referred to the web version of this article.)

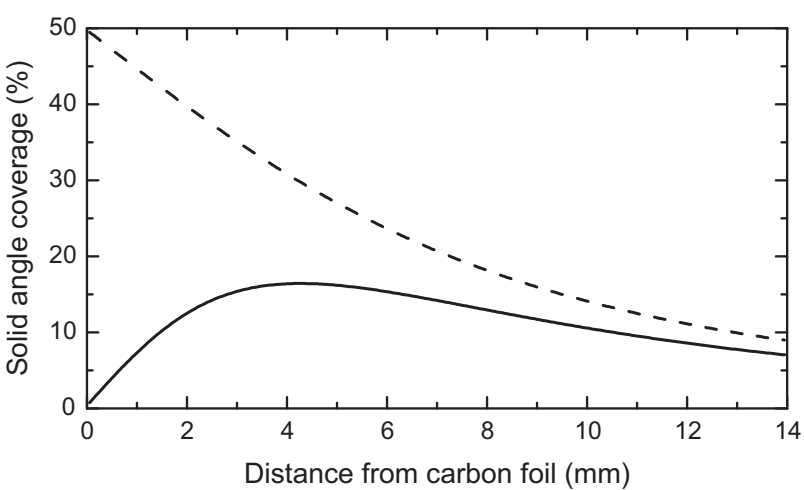

Fig. 4. The calculated solid angle coverage is shown with respect to the distance between the foil and PIPS detectors. For optimum solid angle coverage with the APIPS detector (solid line), the detector should be placed between 3.9 and $5 \mathrm{~mm}$ from the carbon foil. The dash line shows the solid angle coverage for the PIPS detector.

triggered with no implementation of master triggers. In addition to the detector signals, the logic pulses from the release of the proton pulse and the release from the ISCOOL buncher $[21,22]$ can also be fed into the digital DAQ. These logic signals can then be used during offline analysis for correlating the arrival of the ion bunches in the DSS to the decay events measured by the silicon detectors. The DGF DAQ is a modular unit. The number of channels can be increased by adding DGF modules to the DAQ system.

\section{Commissioning with ${ }^{221} \mathrm{Fr}$}

\subsection{Setup}

The francium isotopes were produced at the ISOLDE facility by impinging $1.4 \mathrm{GeV}$ protons upon a UCx target. The atoms were ionized on a rhenium surface and extracted from the ion cavity. They were then accelerated to $30 \mathrm{keV}$ and mass selected using the high-resolution mass separator. The radio-frequency quadrupole Paul trap (ISCOOL) [22] was utilized to cool and bunch the ions before being delivered to the CRIS beam line and ultimately to the DSS.

A 90\% high-purity germanium detector (Model: GC9019-79357) was placed beside the DSS chamber at the on-beam axis location. The efficiency of this detector was measured for $\gamma$-rays emitted at the location of the carbon foil using three sources: ${ }^{133} \mathrm{Ba},{ }^{137} \mathrm{Cs}$ and ${ }^{152} \mathrm{Eu}$. These $\gamma$-ray sources could not be placed in the exact position of the foil due to spatial constraints. Instead, the sources were attached individually to both the nearside and far-side locations of each detector. This allowed for a maximum and minimum detection efficiency to be calculated for each $\gamma$-ray energy, as shown in Fig. 5. The presence of the PIPS and APIPS detectors was also accounted for in the efficiency measurement.

The energy resolution (FWHM) of the silicon detectors was investigated using a quadruple $\alpha$ source $\left({ }^{148} \mathrm{Gd},{ }^{239} \mathrm{Pu},{ }^{241} \mathrm{Am}\right.$, ${ }^{244} \mathrm{Cm}$ ) placed at the position of a carbon foil. For comparison, both an analog DAQ system as well as the digital DAQ were used for this measurement. The results for both systems were consistent with a FWHM value of $18 \mathrm{keV}$ (at $5.485 \mathrm{MeV} \alpha$-decay from ${ }^{241} \mathrm{Am}$ ) for the PIPS detector and $19 \mathrm{keV}$ for the APIPS detector. These values agree favorably with the manufacturer's specifications (17 keV for the PIPS and $18 \mathrm{keV}$ for the APIPS) given in Section 2.1. During the online experiment, the observed resolution of the PIPS detector was less than the APIPS. This loss of resolution was explained by the energy straggling of $\alpha$ particles traveling through a larger thickness of the carbon foil to get to the PIPS detector.

\subsection{Results}

Decay spectroscopy of ${ }^{221} \mathrm{Fr}$ was performed for commissioning the DSS. The radioactive beam was implanted on a carbon foil with the PIPS and APIPS detectors situated on either side of it. The $\alpha$-particle energy spectrum obtained from these detectors, following the decay of ${ }^{221} \mathrm{Fr}$ and its daughter nuclei, is shown in Fig. 6(a). $\alpha$-Rays subsequent to the $\alpha$ decay were measured in the germanium detectors and $\alpha-\gamma$ coincidences were also made possible by time correlating the time-stamped data. Fig. 6(b) demonstrates the coincidence measurement showing a $218 \mathrm{keV} \gamma$-ray peak gated on the $6126 \mathrm{keV} \alpha$-energy peak and a $1 \mu \mathrm{s}$ coincidence timing window applied. These data successfully commissioned the DSS.

\section{Summary}

A new UHV-compatible decay spectroscopy station has been built and is dedicated to the CRIS beam line. Tests were conducted to determine the $\gamma$-ray efficiency of the DSS and the extent the 


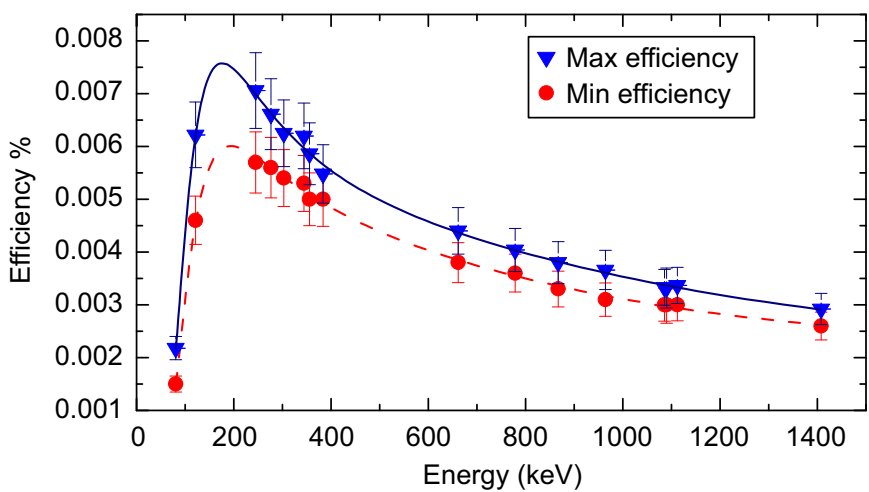

Fig. 5. Minimum (circle) and maximum (triangle) efficiency curve for the $90 \%$ high purity germanium detector. The difference in the efficiency is due to the location of the source placed on the near side (maximum) and far side (minimum) of the carbon foil.

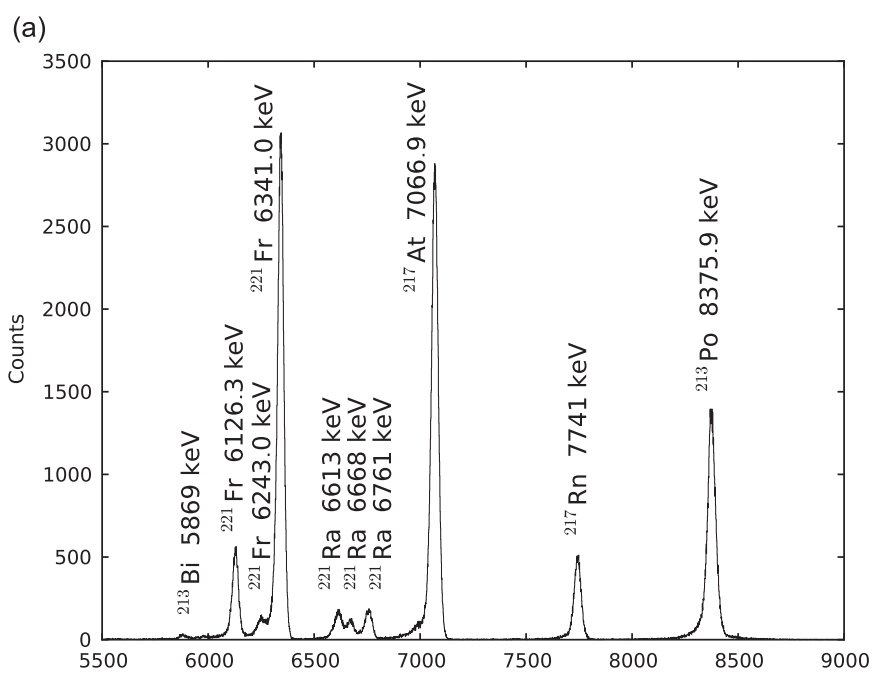

(b)

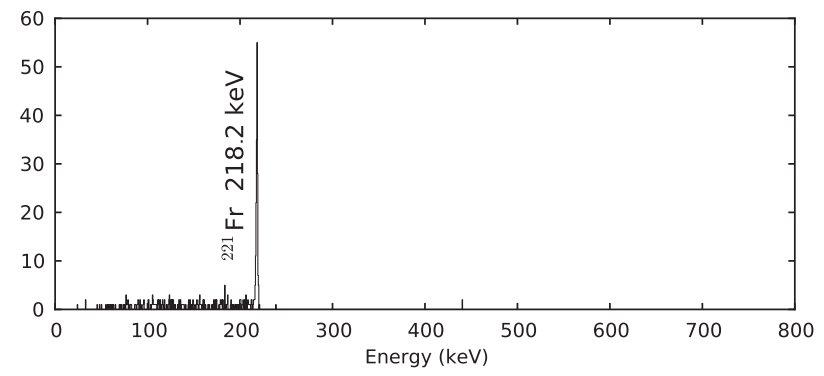

Fig. 6. (a) Alpha-decay energy spectrum of ${ }^{221} \mathrm{Fr}$ and its daughter nuclei. (b) Gamma-energy spectrum showing the result of an $\alpha-\gamma$ coincidences gate applied on the $6126 \mathrm{keV} \alpha$-decay of ${ }^{221} \mathrm{Fr}$. The $218 \mathrm{keV} \gamma$-ray from the fine structure is indicated.

attenuation by the steel chamber and other components. At energies higher than $591 \mathrm{keV}$, an effect of less than $18 \%$ was observed but at lower energies this was more prominent with approximately $92 \%$ at $59 \mathrm{keV}$ and $52 \%$ at $123 \mathrm{keV}$. The attenuation by the steel wheel is an advantage since it masks the $\gamma$-rays emitted from the carbon foils containing previously implanted ions different from those that are being measured.

The DSS was commissioned using radioactive ${ }^{221} \mathrm{Fr}$ beams transported through the CRIS beam line and implanted in a carbon foil on the windmill system. The $\alpha$ and $\gamma$ spectra were collected and analyzed. As a proof-of-principle, $\alpha-\gamma$ coincidences were observed following the $\alpha$ decay of ${ }^{221} \mathrm{Fr}$, successfully demonstrating the operation of the DSS.

\section{Acknowledgements}

The authors extend their thanks to the ISOLDE team for providing the beam, the GSI target lab for producing the carbon foils, IKS-KU Leuven and University of Manchester machine shops for their work, IKS-KU Leuven and the UK-France loan pool for the use of their Ge detectors. This work was supported by the IAPproject P6/23 of the OSTC Belgium (BRIX network) and by the FWO-Vlaanderen (Belgium). The Manchester group was supported by the STFC consolidated grant ST/F012071/1 and continuation grant ST/J000159/1. M.M. Rajabali was funded through the P6/23 project and partially by the Natural Sciences and Engineering Research Council of Canada. K.T. Flanagan was supported by STFC Advanced Fellowship Scheme grant number ST/F012071/1. The University of Surrey support was from STFC grant ST/J000051/1 and AWE plc. The authors would also like to thank Ed Schneiderman for continued support through donations to the Physics Department at NYU.

\section{References}

[1] Yu. Kudryavtsev, et al., Nuclear Instruments and Methods in Physics Research Section B 204 (2003) 336.

[2] U. Köster, V. Fedoseyev, V. Mishin, Spectrochimica Acta B 58 (2003) 1047.

[3] C. Rauth, et al., Nuclear Instruments and Methods in Physics Research Section B $215(2004) 268$.

[4] I.D. Moore, et al., Journal of Physics G 31 (2005) S1499.

[5] K. Blaum, et al., Nuclear Instruments and Methods in Physics Research Section B 204 (2003) 331.

[6] T. Sonoda, et al., Nuclear Instruments and Methods in Physics Research Section B 267 (2009) 2918.

[7] L. Weissman, et al., Physical Review C 65 (2002) 024315.

[8] T.E. Cocolios, et al., Journal of Physics G 37 (2010) 125103.

[9] I. Stefanescu, et al., Physical Review Letters 98 (2007) 122701.

[10] J. Van Roosbroeck, et al., Physical Review Letters 92 (2004) 112501.

[11] B. Cheal, K.T. Flanagan, Journal of Physics G 37 (2010) 113101.

[12] J. Billowes, et al., CERN-INTC-2008-010, INTC-P-240, CERN, Geneva, 2008.

[13] T. Procter, et al., Journal of Physics: Conference Series 381 (2012) 012070

[14] P. Dendooven, Ph.D. Thesis, Katholieke Universiteit Leuven, 1992.

[15] T.E. Cocolios, Ph.D. Thesis, Katholieke Universiteit Leuven, 2010.

[16] H.D. Witte, et al., European Physical Journal A 23 (2005) 243.

[17] A.N. Andreyev, et al., Physical Review Letters 105 (2010) 252502.

[18] H.D. Witte, et al., Physical Review Letters 98 (2007) 112502.

[19] B. Lommel, et al., Nuclear Instruments and Methods in Physics Research Section A 480 (2002) 199.

[20] W. Hennig, et al., Nuclear Instruments and Methods in Physics Research Section B 261 (2007) 1000.

[21] H. Franberg, et al., Nuclear Instruments and Methods in Physics Research Section B 266 (2008) 4502

[22] E. Mané, et al., European Physical Journal A 42 (2009) 503. 